Reprod. Nutr. Dévelop., 1980, 20 (5 B), 1701-1706.

\title{
Rôle de cing souches de lactobacilles sur la dégradation des glucides chez le poulet monoxénique
}

\author{
par Odette SZYLIT, Martine CHAMP*, Nadra AIT-ABDELKADER, P. RAIBAUD \\ Laboratoire d'Ecologie Microbienne, INRA \\ 78350 jouy en Josas, France. \\ * Laboratoire de Technologie des Aliments des Animaux, INRA \\ Chemin de la Géraudière, 44072 Nantes Cedex, France.
}

Summary. Role of five Lactobacillus strains on carbohydrate degradation in monoxenic chickens.

Five strains of Lactobacillus were isolated in holoxenic roosters ; two of these which had an $\alpha$-amylase were inoculated separately into 5 groups of axenic chickens fed the same diet. Some differences among these 5 groups were noted. Lactobacillus proliferation varied between ten and a thousand-fold, depending on the strain, and for the same strain depending on whether the crop, caecum or faeces was examined. Amylolytic lactobacilli in vivo played a role in starch degradation in various ways related to the specific properties of their amylase. Lactic acid production in the crop was higher with the three strains producing the two lactic acid isomers than with the two strains producing only one of the isomers. Finally, monoxenic caecal digestion was different from that of both the axenic and the holoxenic.

\section{Introduction.}

Chez le poulet axénique, la digestion glucidique diffère de celle observée en présence d'une flore d'holoxénique. Dans le jabot, la dégradation de l'amidon en oligoholosides dépend de systèmes enzymatiques complexes fournis d'une part par l'hôte ef d'autre part par ses bactéries et la fermentation des oligoholosides en acides $D$ et $L$ lactiques se fait exclusivement sous l'action des lacticodéshydrogénases bactériennes. Bien que possédant un équipement enzymatique important à différents niveaux digestifs, le poulet axénique ne peut dégrader complètement l'amidon : ses contenus cæcaux renferment une forte concentration de glucides (16 p. 100 MS) représentant l'ensemble des produits de la dégradation de l'amidon (lvorec-Szylit, Raibaud et Schellenberg, 1973) alors que chez l'animal holoxénique, la dégradation de l'amidon est terminée quand le bol alimentaire atteint le duodénum et on ne trouve dans les cæca que des traces de glucose.

C'esł pourquoi, nous avons recherché dans quelle mesure les lactobacilles, qui constituent la flore dominante du jabot chez les holoxéniques interviennent spécifiquement sur l'amylolyse et les fermentations lactiques. Pour cela, nous avons suivi chez 5 groupes de poulets monoxéniques hébergeant chacun une souche de lacto- 
bacilles différente, l'établissement de ces souches et la digestion postprandiale des glucides au niveau du jabot et des cæca.

\section{Matériel et méthodes.}

Souches de lactobacilles. - Les cinq souches étudiées ont été isolées du jabot de coqs holoxéniques. Parmi les 3 souches (LEM 220, 207 et 206) produisant les acides D ef $L$ lactiques, les deux premières sont amylolytiques. In vitro (Champ, 1978) leurs $\alpha$-amylases se distinguent par leur $\mathrm{pH}$ optimum d'action (respectivement 5,5 et 6,8 ) ef les produits de dégradation de la souche 207 sont plus polymérisés que ceux de la souche 220. Les deux autres souches utilisées (LEM 202 et 204) donnent in vitro la première 90 p. 100 de $D$ lactate et 10 p. 100 de $L$ tandis que la seconde ne produit que du $\mathrm{L}$ lactate. Les 5 souches appartiennent au sous-genre Thermobacterium.

Obtention des poulets monoxéniques. - Les poussins nés axéniques dans des isolateurs en polychlorure de vinyl (Le Coz, Szylit et Ducluzeau, 1977) sont, soit laissés dans cet état, soit ensemencés per os, le deuxième ou le troisième jour, avec l'une des souches de lactobacilles (poussins monoxéniques) ou avec la flore complexe de poulets holoxéniques (poussins holoxénisés).

Aliment. - L'aliment est un régime semi-synthétique granulé (MD 03) à base d'amidon de maïs (65 p. 100) et de farine de poisson ( 23 p. 100). Il contient par ailleurs 3 p. 100 de maltodextrines qui, d'après des expériences préliminaires, sont nécessaires à l'établissement des souches 220 et 206. Il est stérilisé par irradiation $\gamma$ à 4 Mrad.

Prélèvements ef analyse des contenus digestifs. - Les contenus de jabot et de cæca des poulets monoxéniques 220, 207 et 206 sont prélevés après abattage. Les poulets monoxéniques 202 et 204 sont fistulés au niveau du jabot et les prélèvements des contenus de cet organe sont effectués in vivo à des temps variant de $1 \mathrm{~h} 30$ à $6 \mathrm{~h}$ après le début du repas.

On prélève stérilement dans le jabot et les cæca environ $250 \mathrm{mg}$ de contenu que l'on ensemence dans le milieu GAPT g 10 (Raibaud et al., 1973) en utilisant la technique des dilutions décimales. On mesure ensuite le $\mathrm{pH}$ à l'aide d'une micro-électrode. Enfin on prélève le reste du contenu pour l'analyse biochimique. Sur les contenus des animaux sacrifiés et sur ceux prélevés dans le jabot des poulets fistulés, on effectue l'extraction ef le dosage des glucides solubles ef des acides $D$ et $L$ lactiques selon des méthodes décrites par ailleurs (lvorec-Szylit et Szylit, 1965 ; Szylit, Delort-Laval et Borgida, 1974).

\section{Résultats.}

1. Etablissement des lactobacilles chez le poulet axénique.

Dans les fèces, avec les trois souches 220, 207 et 206 produisant les deux isomères de l'acide lactique, on obtient dès la première semaine qui suit l'ensemencement per os de la souche, un niveau d'établissement variant entre $10^{8}$ ef plus de $10^{9}$ bactéries $/ \mathrm{g}$ de fèces fraîches. 
Avec la souche L lactique 204 l'établissement se fait à un niveau comparable à celui observé précédemment mais beaucoup plus lentement. Par contre avec la souche D 202 non seulement l'établissement est très lent, mais le nombre de bactéries est toujours faible : $10^{5}$ bactéries/g de fèces fraîches, un mois après l'ensemencement.

Les examens post mortem sur les différents groupes de monoxéniques abattus entre 3 et 5 semaines d'âge montrent que les dénombrements au niveau cæcal faits $3 \mathrm{~h}$ après le début du repas sont comparables à ceux effectués dans les fèces : $10^{5}$ bactéries/g de contenu frais pour la souche $202,10^{8}$ bactéries/g pour les autres groupes.

Dans le jabot des poulets sacrifiés ou porteurs d'une fistule, les niveaux d'établissement des différentes souches sont comparables : $10^{8}$ bactéries $/ g$ de contenu pour les souches 207 ef 202 et $10^{7}$ pour les 3 autres souches (206, 220 et 204). On voit donc que la souche 202 ne s'établit à un niveau élevé que dans le premier compartiment digestif du poulet.

2. Rôle des lactobacilles amylolytiques dans la dégradation des glucides aux niveaux du jabot et des cæca.

Dans le jabot, les amylases des lactobacilles interviennent dans la dégradation de l'amidon (fig. 1a). Avec le monoxénique hébergeant la souche non amylolytique 206 , on observe que le schéma de la dégradation des glucides se rapproche de celui de l'axénique : la concentration en glucides à courtes chaînes n'évolue pas entre la $3^{\mathrm{e}}$ et la $6^{\mathrm{e}}$ heure postprandiale. Par contre le processus d'amylolyse est le même chez le monoxénique hébergeant la souche amylolytique 220 et chez l'holoxénisé. Si l'on compare les deux groupes de poulets monoxéniques hébergeant une monoflore amylolytique différente, on constate que les processus d'amylolyse dans le jabot diffèrent selon la souche établie. Au cours des 3 premières heures postprandiales la dégradation de l'amidon est plus rapide avec la souche 207 qu'avec la souche 220 comme en témoigne la teneur en amidon restante : 26,7 p. 100 contre 33,7 p. 100 du contenu lyophilisé. La dégradation se poursuit entre $3 \mathrm{~h}$ et $6 \mathrm{~h}$ seulement avec la souche 220 : en effet, la teneur en amidon passe de 33,7 p. 100 à 16,9 p. 100 chez le poulet 220 , alors qu'elle est encore de 23,2 p. 100 chez le pouleł 207,6 h après le repas. Des différences importantes apparaissent dans la concentration en glucides alcoolosolubles $G_{1}-G_{14}$ des contenus de jabot des 2 groupes de poulets : respectivement 7,7 et 5,5 p. 100 du contenu lyophilisé, pour le poulet 220 ef $207,6 \mathrm{~h}$ après le repas ; de plus le rapport entre les glucides $G_{1}-G_{4}$ et les glucides $G_{1}-G_{14}$ est alors de 85,4 p. 100 avec la souche 220 et de 65,3 p. 100 avec la souche 207 . A l'inverse la concentration en glucides plus polymérisés est moins élevée avec la souche 220 qu'avec la souche 207 : respectivement 16,5 p. 100 et 24,5 p. 100 du contenu lyophilisé, $6 \mathrm{~h}$ après le repas.

Dans les cæca, on trouve une même quantité de glucides solubles avec les 3 souches productrices d'acides $D$ et $L$ lactiques. La présence de ces glucides solubles différencie les sujets monoxéniques 220 et 207 à la fois des holoxénisés qui n'ont plus de glucides fermentescibles à ce niveau, et des axéniques qui en possèdent une plus forte proportion (fig. 1a).

De même, l'évolution quantitative et qualitative des glucides hydro- ef alcoolosolubles du contenu cæcal entre 3 et $6 \mathrm{~h}$ après le début du repas est peu différente chez les deux groupes monoxéniques hébergeant les monoflores amylolytiques 220 et 207. 
Jabot
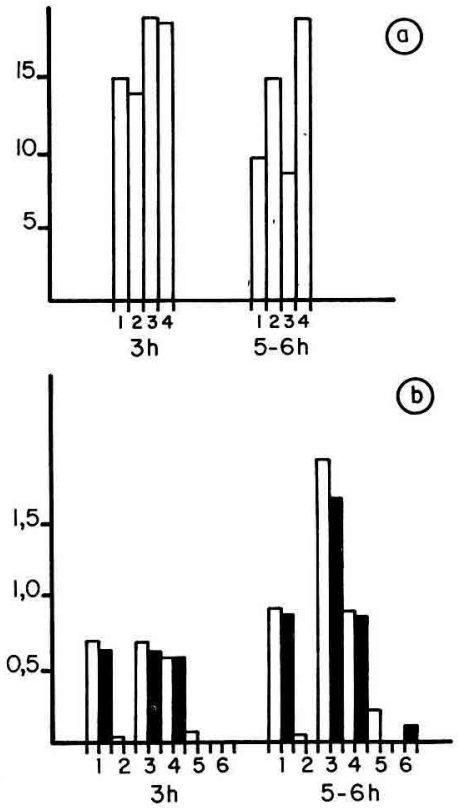

Caeca
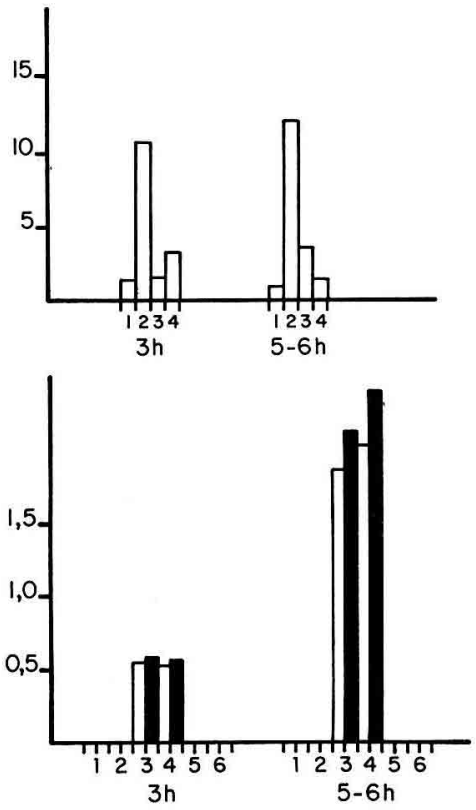

FIG. 1. - Evolution des glucides solubles à courtes chaines et de l'acide lactique dans le jabot ef les cæca des poulets ax- holo- ef monoxénique. a) glucides solubles (p. 100 d'amidon); b) acide lactique (p. 100 matière sèche). 1. - Holoxénique; 2. - Axénique; 3. - Monoxénique 220 (souche amylolytique) ; 4. - Monoxénique 206 (souche non amylolytique); 5. - Monoxénique 204 (souche L lactique) ; 6. - Monoxénique 202 (souche D lactique). a L lactique ; - D lactique.

Les concentrations de glucides alcoolosolubles (15,9 et 17,0 p. 100 du contenu lyophilisé, $6 \mathrm{~h}$ après le repas) demeurent supérieures à celles des hydrosolubles $(8,5$ et 7,7 p. 100). Qualitativement on retrouve $G_{1}, G_{2}$ ef $G_{3}$ dans les cæca des monoxéniques comme dans ceux des animaux axéniques. Les animaux monoxéniques diffèrent des axéniques par la présence dans leur cæca d'un cinquième composé glucidique qui migre entre le glucose et le maltose.

3. Rôle des différentes souches de lactobacilles sur la production d'acide lactique dans le jabot et les cæca (fig. 1b).

Dans le jabot, avec les souches 206 et 220 produisant les 2 isomères, in vivo comme in vifro on observe la formation d'acides $D$ ef $L$ lactiques en concentration égale, que la bactérie ne soit pas (206) ou soit (220) amylolytique et cette production comme chez l'holoxénique apparaît entre la $3^{\mathrm{e}}$ et la $6^{\mathrm{e}}$ heure postprandiale. Ces métabolites sont absents du jabot des axéniques. Avec la monoflore 206, la production d'acides D et $L$ lactiques formés à partir des glucides fermentescibles présents dans l'aliment ou provenant de la dégradation de l'amidon par l'amylase salivaire est relativement faible. Avec la monoflore 220, elle est deux fois plus importante que chez l'holoxénisé $(1,7$ et 1,6 p. 100 contre 0,9 et 1,0 p. 100) et le $\mathrm{pH}$ est plus bas $(4,6$ contre 5,1$)$. 
Avec les deux souches ne produisant qu'un seul des 2 isomères, la formation d'acide lactique a été suivie en cinétique sur les 3 animaux porteurs d'une fistule. Là encore, la formation est maximum 6 h après le début du repas mais les concentrations en acide lactique restent très faibles ef les $\mathrm{pH}$ évolvent peu.

In vivo comme in vitro, avec la souche 202 , il se forme essentiellement de l'acide D lactique et des traces de $L$. Les concentrations maximum sont comprises entre 0 et $1 \mathrm{mg} / \mathrm{g}$ de matière fraîche et le $\mathrm{pH}$ reste voisin de 6. De même avec la souche 204, il se forme in vivo comme in vitro essentiellement $d u L$ lactate, cette formation est peu importante $(2 \mathrm{mg} / \mathrm{g}$ de matière fraîche $5 \mathrm{~h}$ après le début du repas). Le D lactate ne se trouve que sous forme de traces et n'évolue pas au cours du temps. Les valeurs de $\mathrm{pH}$ se situent entre 5,44 et 5,72 à l'exception d'un cas où le $\mathrm{pH}$ était de 5,16.

Dans les cæca des monoxéniques 220 et 206, il se forme de l'acide DL lactique en quantité égale ef importante chez les deux groupes (environ 2,5 p. 100 de chaque isomère). Il résulte de cetfe fermentation lactique un $\mathrm{pH}$ plus bas $(5,1)$ avec les monoflores qu'avec une flore d'holoxénisé $(\mathrm{pH}=5,4)$ et que chez l'axénique $(\mathrm{pH}=6,2)$.

II n'y a pas formation d'acide lactique dans les cæca des monoxéniques 204 et 202.

\section{Discussion et conclusion.}

De l'ensemble des résultats, on peut observer un certain nombre de différences dans le niveau d'établissement des souches de lactobacilles et dans la physiologie digestive des différents groupes de poulets monoxéniques.

L'établissement des bactéries varie à la fois avec la souche de lactobacilles étudiée et pour une même souche avec le compartiment digestif. Le jabot paraît être le compartiment le plus stable où la population de lactobacilles se multiplie bien. Dans les cæca et les fèces le niveau d'établissement pourrait être fonction des substrats glucidiques encore disponibles dans la dernière partie du tube digestif. Ainsi des examens en microscopie électronique à balayage (Gallant, non publié) montrent que les fèces du monoxénique 207 , où l'on comple $10^{9}$ bactéries/g de fèces fraîches, renferment de nombreux grains d'amidon modifiés alors que l'on compte seulement $10^{6}$ bactéries dans celles du monoxénique 220 ne contenant plus d'amidon. Chez le poulef le niveau d'implantation des lactobacilles dans le jabot est donc toujours supérieur ou égal à celui des cæca ou des fèces, alors que le contraire s'observe dans le cas des souris monoxéniques (Ducluzeau ef Raibaud, 1969).

Dans le jabot, les lactobacilles amylolytiques 220 et 207 participent à la dégradation des glucides avec des modalités variables liées aux propriétés spécifiques de leur amylase. Chez le monoxénique 220 , le processus d'amylolyse et de fermentation lactique se rapproche de celui de l'holoxénisé. Le monoxénique 207 se différencie du 220 par un processus d'amylolyse et de fermentation lactique extrêmement rapide.

La production d'acide lactique est également variable selon la souche établie. On remarque qu'elle est plus importante avec les 3 souches produisant les 2 isomères de l'acide lactique qu'avec les 2 autres souches produisant uniquement de l'acide $L$ ou D lactique sans, pour autant, que l'on puisse faire une relation de cause à effet entre production d'acide lactique ef nature de l'isomère produit. Chez les monoxé- 
niques et les holoxénisés comme chez les holoxéniques (Bolton, 1965 ; Ivorec-Szylit ef Szylit, 1965) les concentrations en acide lactique ne deviennent importantes que $6 \mathrm{~h}$ après le début du repas. Du fait de cette similitude on peut penser que les résultats obtenus sur poulets monoxéniques reflètent, pour une large part, les phénomènes se passant chez des animaux holoxéniques où ces lactobacilles vivent en association avec un grand nombre d'autres souches bactériennes.

La digestion cæcale des monoxéniques se différencie à la fois de celles des holoxénisés, qui n'ont plus de glucides fermentescibles à ce nivequ digestif, et de celles des axéniques, qui ont comme les monoxéniques, de fortes concentrations de glucides mais pas d'acide lactique.

L'interprétation physiologique des relations microflore digestive glycolytiquenutrition est encore partielle comple tenu de la méthodologie extrêmement longue ef du faible nombre d'animaux mis en jeu à chaque essai. Cependant, ces recherches entreprises conjointement avec des études de rétentions énergétique et azotée et d'adsorption permettront d'estimer pour le poulet l'importance du potentiel amylasique des lactobacilles et la valeur nutrition nelle de l'acide lactique, plus particulièrement celle de l'isomère $D$ qui est mal utilisé par les tissus des animaux supérieurs et dont le rôle physiologique est préjugé néfaste.

Journées Ingestion-Digestion-Absorption de l'Association française de Nutrition Paris, 15-16 novembre 1979.

Remerciements. - Nous remercions Monsieur Léglise d'avoir mis au point et assuré la fistulation des poulets en isolateurs.

\section{Références}

BOLTON W., 1965. Digestion in the crop of the fowl. Br. Poult. Sci., 6, 97-102.

CHAMP M., 1978. Rôle de deux lactobacilles amylolytiques dans la dégradation de l'amidon chez le poulet monoxénique. Th. 3e Cycle, Orsay no d'ordre 2532.

DUCLUZEAU R., RAIBAUD P., 1969. Ensemencement de douze souches bactériennes dans le tube digestif de souris axéniques. Il. Equilibre obtenu entre ces souches dans différents segments du tube digestif. Ann. Inst. Pasteur, 116, 370-382.

IVOREC-SZYLIT O., SZYLIT M., 1965. Contribution à l'étude de la dégradation des glucides dans le jabot du coq. Mise en évidence ef dosage des stéréoisomères $D$ ef $L$ lactate. Ann. Biol. anim. Bioch. Biophys., 5, 353-360.

IVOREC-SZYLIT O., RAIBAUD P., SCHELLENBERG P., 1973. Breakdown of starch at different levels of digestive tract in the germ-free and the conventional chicken, 225-231. In HENEGHAN J. B., Germ free research : Biological effect of gnotobiotic environments. Acad. Press. N. Y., London.

LE COZ Y., SZYLIT O., DUCLUZEAU R., 1977. Mise au point des isolateurs de plastique souple pour des expériences nutritionnelles pour poulet axénique et gnotoxénique. Sci. Tech. Anim. Lab., 2, 27-33.

RAIBAUD P., GALPIN J. V., DUCLUZEAU R., MOCQUOT G., OLIVER G., 1973. Le genre Lactobacillus dans le tube digestif du rat. I. Caractères des souches hormofermentaires isolées de rats holo- et gnotoxéniques. Ann. Microbiol. Inst. Pasteur, 124A, 83-109.

SZYLIT O., DELORT-LAVAL J., BORGIDA L. P., 1974. Dégradation dans le jabot du coq ef efficacité d'amidons de maïs à différents taux d'amylose sur la croissance du poulet. Ann. Zootech., 23, 253-265. 